\title{
Surgical Results of Non-small Cell Lung Cancer in Nepal
}

\author{
Binay Thakur, ${ }^{1}$ Di Yonghui, ${ }^{2}$ Mukti Devkota, ${ }^{1}$ Paribartan Baral, ${ }^{1}$ Bishowram Poudel ${ }^{1}$ \\ 'Department of Surgical Oncology, B. P. Koirala Memorial Cancer Hospital, Nepal
}

\section{ABSTRACT}

Introduction: Surgery remains the only curative modality for early stage of Non small cell lung caner (NSCLC). We reviewed overall scenario of lung cancer and surgical results in Nepal.

Methods: 1000 patients with the pathological diagnosis of lung cancer were analyzed to summarize the overall scenario of lung cancer. 157 NSCLC patients underwent pulmonary resection. CIA, IB, IIA, IIB, IIIA (N1) underwent surgery as initial modality of treatment. cIIIA (N2) patients were taken for surgery after neoadjuvant chemo/ chemoradiation.

Results: Analysis of 1000 patients showed squamous cell carcinoma in 41.3\%, incurable stage (IIIB/ IV) in $66.8 \%$ and rate of curative resection in $6.7 \%$ cases. Surgery as a single modality of treatment was used in $38 \%$. Neoadjuvant Chemo/ chemoradiation/ radiation followed by surgery and surgery followed by chemo/ chemoradiation/ radiation was done in 12\% and 50\%, respectively. In-hospital mortality was $2 \%$ (post pneumonectomy: $5.5 \%$; post lobectomy: $1.5 \%$; post sublobar resection: $0 \%$ ). $\mathrm{R} 0$ resction was achieved in 91\% cases. Median survival and 5-year overall survival were 36 months and $18 \%$, respectively. Better survival was achieved in pI-II vs pIII/ IV, pN0-1 vs pN2 and pR0 vs $\mathrm{pR}+(\mathrm{p}<0.05)$

Conclusions: Earlier stage (pI-II), R0 resection and pathological pNo-1 has the best five year overall survival in Nepalese patients with NSCLC as well.

Keywords: lung cancer; NSCLC; pulmonary resection.

\section{INTRODUCTION}

Lung cancer is the most common cause of cancer death both in male and female. More than 1.35 million people die of lung cancer annually worldwide. ${ }^{1-4}$

Lung cancer is often diagnosed in advanced stage in older individuals who often harbor additional comorbidities, rendering most patients unresectable and incurable. Approximately $85 \%$ of lung cancers are Nonsmall cell lung cancer (NSCLC) and surgery remains the only curative modality of treatment. But only $25-30 \%$ of these patients are suitable for surgery. ${ }^{4}$

Improving the survival of NSCLC patients is a major challenge for modern multimodality oncological treatment strategies, considering that the 5-year survival remains less than $15 \%$ across all stages of disease with fewer than $7 \%$ of patients alive 10 years after diagnosis. ${ }^{5}$

For medically operable patients, lobectomy, the surgical resection of a single lobe, with mediastinal lymph node dissection (MLND) or systematic lymph node sampling (SLNS), is generally accepted as the optimal procedure

Correspondence: Dr Binay Thakur, Department of Surgical Oncology, B.P. Koirala Memorial Cancer Hospital, Bharatpur, Nepal. E-mail: binaythakur@hotmail.com, Tel: +977-9855055931. 
for early tumor stage..$^{6,7}$

Scenario of lung cancer and surgical results of NSCLC have never been properly studied in Nepal. The aim of this article was to perform a retrospective analysis of lung cancer patients, assess the resectibility rate and to determine long term survival.

\section{METHODS}

A retrospective analysis of 1000 patients with the diagnosis of lung cancer was performed in order to summarize the overall scenario of lung cancer in Nepal. Clinical staging was done on the grounds of clinical examination, CT chest - abdomen and bronchoscopic findings. NSCLC patients with CIA, IB, IIA, IIB and IIIA (N1) were subjected to surgical treatment as an initial modality. clllA (N2) patients received neoadjuvant chemo/ chemoradiation followed by surgery. Only these later patients $(n=157)$ undergoing surgical resection were reviewed in detail.

\section{Mediastinal nodal staging}

Nodal status was judged on the basis of CT Chest and infrequently by mediastinoscopy ( 3 patients/ $2 \%$ ). Ipsilateral mediastinal node more than $1 \mathrm{~cm}$ in diameter along its short axis was assumed to have N2 disease. PET scan (done abroad) was used as a staging tool in 5 patients $(3 \%)$.

\section{Fitness for surgery}

Patients were taken for surgery only when the following conditions were met:

1. ECOG: $0-2$

2. $\mathrm{SPO} 2$ at room air $>90 \%$

3. Baseline FEV $1>75 \%$

4. Predicted post op FEV $1>40 \%$ (using spirometry)

5. Desaturation by $<4 \%$ after brisk 6 minute walk test or after 20 sit-up

6. Left ventricular ejection fraction $>60 \%$

\section{Surgical approach}

Patients were conventionally approached using postero-lateral thoracotomy through $5^{\text {th }}$ intercostal space. Selected patients with cT1-2 underwent VATS lobectomy. For VATS, one $10 \mathrm{~mm}$ optical port, two $5 \mathrm{~mm}$ working ports and a 4-6 $\mathrm{cm}$ utility incision were used. Nodes were either sampled randomly, systematically sampled or completely dissected. In random sampling, only suspected (on visual and tactile inspection) were randomly sampled. In systematic sampling, three mediastinal stations were sampled. During mediastinal nodal dissection, levels $1,2 \mathrm{R}, 3,4 \mathrm{R}$, $7,8,9,10-13$ were excised for right sided tumors. For left sided tumors, levels 5, 6, 7-13 were excised.

Vessels were manually ligated or tansfixed before division. Bronchus was either stapled or stump was sutured manually with absorbable polygalactin 3-0 suture.

\section{Adjuvant treatment}

After RO resection, post operative adjuvant treatment was rarely used (before year 2010). After R1/ R2 resection, patient received adjuvant radiation/ chemoradiation.

Since year 2010, after RO resection all the patients above pIB and selected pIB with poor prognostic factors received platinum based chemotherapy. pN2 patients received post operative radiation as well.

\section{Neoadjuvant treatment}

Patients with cN2 disease received 2-3 cycles of platinum based chemotherapy. After completion of chemotherapy, re-investigation was done and if there was either a response or no progression of disease, patient was taken for surgery. One patient, who had superior sulcus tumor, received preoperative radiation.

\section{Follow-up}

Patients were followed up every 4 months for $1^{\text {st }}$ two years with clinical examination, Chest $X$-ray and USG of abdomen. Annually, CT chest and upper abdomen were advised. From 3-5 years, patients were followed up every six months in the similar manner. If there was any suspicion on disease relapse, radiological investigations were advised accordingly.

\section{Statistical analysis}

Statistical analysis was done using SPSS 16.0 software. Continuous variables were compared using student's t-test. Categorical variables were compared using Chi-square test. Kaplan-Meier curve was used to plot the survival curve and difference in survival was determined using log-rank test. . $P$ value of less than 0.05 was set as statistically significant.

\section{RESULTS}

Summary of results of 1000 patients with lung cancer have been shown in table 1 . 
Thakur et al. Surgical Results of Non-small Cell Lung Cancer in Nepal.

\begin{tabular}{|ll|}
\hline Table 1. Overall summary of 1000 patients. \\
\hline NSCLC & $82.1 \%$ \\
Squamous cell carcinoma & $41.3 \%$ \\
Adenocarcinoma & $10 \%$ \\
Others & $30.8 \%$ \\
SCLC & $17.9 \%$ \\
Central tumors (located up to segmental & $70.3 \%$ \\
bronchi) & $74.8 \%$ \\
Bronchoscopic abnormality & $66.8 \%$ \\
Incurable stage & $36.1 \%$ \\
Stage IIIB & 23.9 \\
Stage IV & $6.8 \%$ \\
Extensive (SCLC) & $6.7 \%$ \\
Curative surgery & \\
\hline
\end{tabular}

157 patients with cl-IIIA NSCLC with mean age of 58 years underwent pulmonary resection and were studied. 140 (89\%) were smokers. The presenting complaints were cough (145/92\%), hemoptysis (98/ $62 \%)$, chest pain $(92 / 59 \%)$ and shortness of breath (86/ 55\%). One patient (0.6\%) was asymptomatic. Preoperative confirmation of NSCLC was achieved in $114(73 \%)$ of cases whereas in $43(27 \%)$ patients, it was established only after surgery. Treatment modality and surgical procedures have been summarized in table 2 and 3 , respectively.

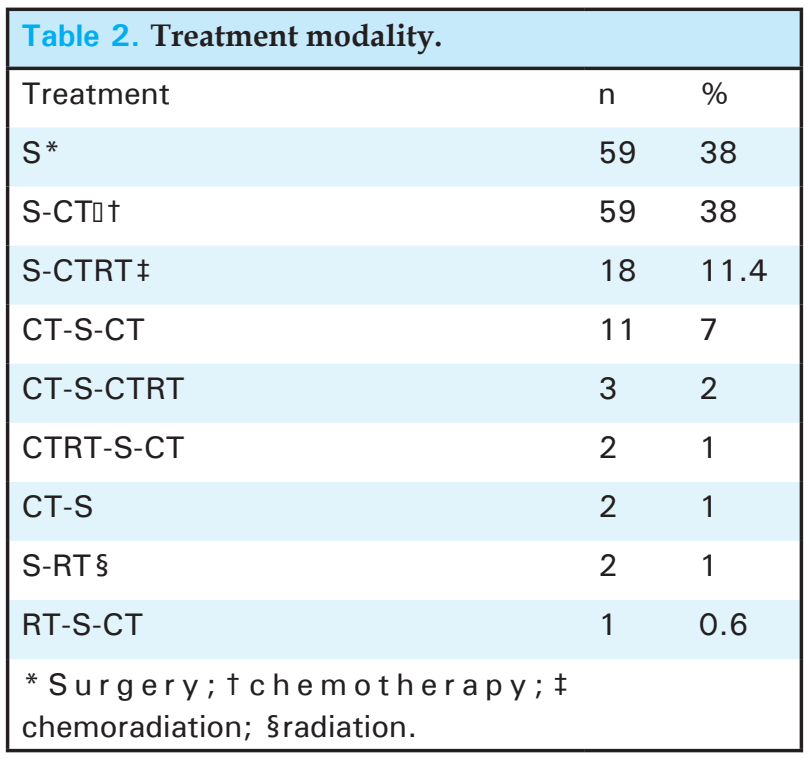

\begin{tabular}{|lll|}
\hline \multicolumn{2}{|l|}{ Table 3. Surgical procedures. } \\
\hline Surgery & $\mathrm{n}$ & $\%$ \\
Pneumonectomy & 18 & 11.5 \\
Lobectomy & 130 & 82.8 \\
Conventional & 120 & 76.4 \\
Sleeve & 10 & 6.4 \\
Bronchial & 8 & \\
Arterial & 2 & \\
Bilobectomy & 7 & 4.5 \\
Segmentectomy & 1 & 0.6 \\
Wedge & 1 & 0.6 \\
\hline
\end{tabular}

VATS lobectomy was attempted in 11 (7\%) cases. Two patients needed conversion due to dense pleural adhesions. Mediastinal nodal dissection, systematic sampling and random sampling were done in 104 (66\%), $51(32 \%)$ and 2 (1\%), respectively. In-hospitality was $2 \%$ (3 patients). Mortality after pneumonectomy, lobectomy and sublobar resection was $5.5 \%$ (1 patient), $1.5 \%$ (2 patients) and $0 \%$. Mean number of dissected nodes was 16 . Mean postoperative stay, intraoperative blood loss and operative time was 10 days, $632 \mathrm{ml}$ and 132 minutes, respectively. RO resection was achieved in $143(91 \%)$ cases.

Final pathological diagnosis has been shown in table 4 .

\begin{tabular}{|lll|}
\hline Table 4. Final histology. & & \\
\hline Type & $\mathrm{n}$ & $\%$ \\
Squamous cell & 116 & 73.8 \\
Adenocarcinoma & 27 & 17.2 \\
Adenosquamous & 5 & 3.2 \\
Large cell & 2 & 1.3 \\
Mucoepidermoid & 2 & 1.3 \\
Adenoca with NED & 2 & 1.3 \\
SCLC* & 2 & 1.3 \\
Carcinoma in situ & 1 & 0.6 \\
\hline
\end{tabular}

${ }^{*}$ Final biopsy revealed Small cell carcinoma.

19 patients received neoadjuvant treatment (NACT/ NACTRT/ NART). Stable, partial response and complete response was found in $42.1 \%, 47.4 \%$ and $10.7 \%$, respectively. Median survival was 36 months with overall 5 -year survival of $18 \%$ (Fig. 1). 
Fig. 1. Overall survival curve.

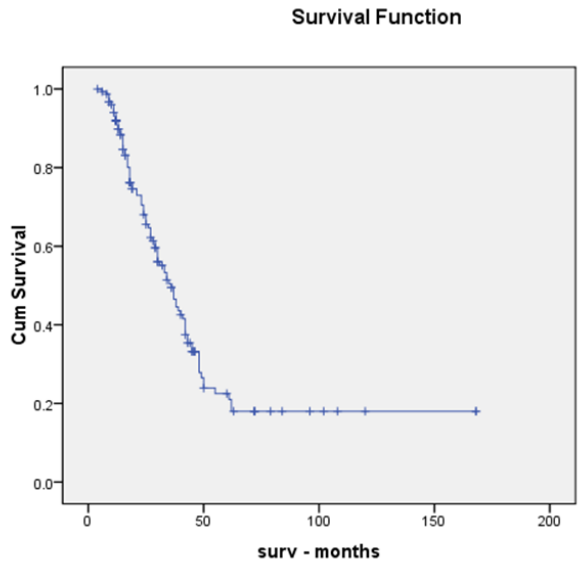

Final pathological stage and stage specific 5-year survival have been shown in table 5 and Figure 2 , respectively.

Table 5. Final pathological stage (UICC $7^{\text {th }}$ edition).

\begin{tabular}{|lccc|}
\hline Stage & $\mathrm{n}$ & $\%$ & $\begin{array}{c}5-y r \text { survival } \\
\text { (\%) }\end{array}$ \\
O & 3 & 1.9 & 100 \\
IA & 4 & 2.5 & 100 \\
IB & 10 & 6.4 & 65 \\
IIA & 18 & 11.5 & 42 \\
IIB & 34 & 21.7 & 29 \\
IIIA & 72 & 45.9 & 18 \\
IIIB & 7 & 4.5 & 0 \\
IV & 9 & 5.7 & 0 \\
\hline
\end{tabular}

Figure 2. Stage specific survival curve.

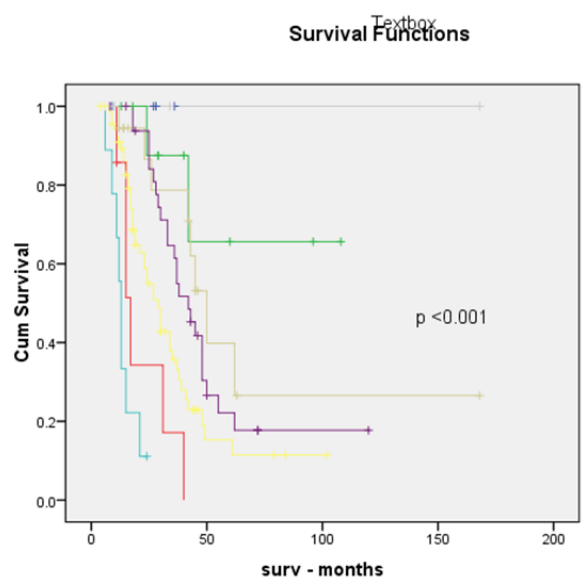

Median survival for $\mathrm{pN} 0, \mathrm{pN} 1, \mathrm{pN} 2$ and $\mathrm{pN} 3$ was 45, 35, 18 and 11 months, respectively. Node specific and resection status specific Survival curves have been shown in Figures 3 and 4 , respectively.

Fig. 3. Node specific survival curve.

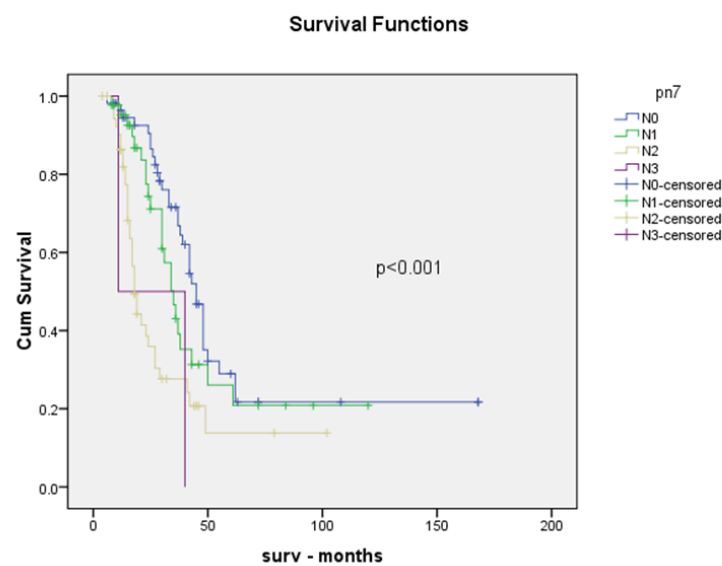

Fig. 4. Resection status and survival.

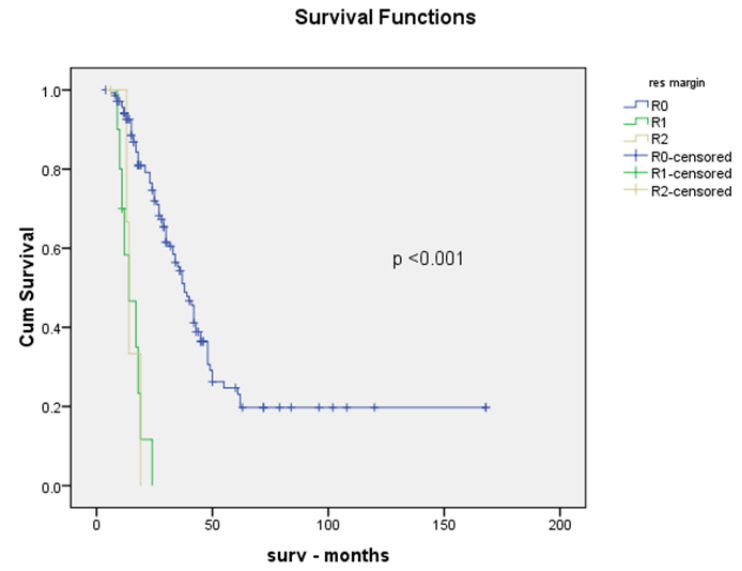

\section{DISCUSSION}

Early stage NSCLC, defined as stage II and II, is usually treated with surgery as an initial modality. ${ }^{8}$ For stage IIIA (N1 disease), surgery again remains the primary modality. Though very controversial, a consensus approach for IIIA (N2 disease) has reached. It is generally divided into three categories with different recommendations: -16 $^{-16}$

1. Unsuspected N2 (detected after surgery): surgery followed by adjuvant chemoradiation is recommended. 
Thakur et al. Surgical Results of Non-small Cell Lung Cancer in Nepal.

2. Preoperatively proven N2, non-bulky: neoadjuvant chemotherapy/ chemoradiation followed by surgery and adjuvant chemoradiation, if the disease doesn't progress after neoadjuvant treatment. Definitive chemoradiation is an alternative option.

\section{Bulky N2: definitive chemoradiation.}

Cerfolio et al reported a 5-year survival rate in patients with unexpected N2 disease who underwent complete resection followed by adjuvant therapy of $35 \%$ while patients with single station N2 disease had even a better outcome. ${ }^{17}$

Though not well studied, Hospital based Cancer incidence data showed lung cancer to be the most common cancer in male and $3^{\text {rd }}$ most common cancer in female in Nepal. ${ }^{18}$ To the best of our knowledge, our study with 1000 lung cancer patients is the only published study till date from Nepal showing squamous cell carcinoma to be the most common type (41.3\%), $66.8 \%$ incurable stages and curative resectional surgical rate of only $6.7 \%$.

From our study it is obvious that fitness for surgery was not assessed according to the established guidelines. The reason for it was the lack of facilities to perform DLCO, ventilation-perfusion scan, and Vo2 max testing. Similarly, diagnostic work up both for nodal and metastatic staging was also suboptimal. FDG-PET has been considered as one of the most important staging tools both for mediastinal nodal evaluation and metastatic work up. Median sensitivity and specificity of FDG-PET were reported as $100 \%$ and $78 \%$, respectively, in patients with enlarged nodes. ${ }^{19}$

Mediastinoscopy (available since last 2 years only), PET scan, endoscopic ultrasound and endobronchial ultrasound were not available in the country.

Despite limitations, our surgical results seem to be quite promising. Out of 157 pulmonary resections, $82.8 \%$ underwent lobectomy, which is the current standard of surgical care. ${ }^{7}$

In the majority of the patients (66\%), complete mediastinal nodal dissection was performed. The number of mean dissected nodes was 16 . RO resection could be achieved in $91 \%$ cases. Our surgical complication rates were also comparable to the results from literature. In a very large study from National Cancer Database (the largest database in the world) from USA $(n=119146)$, overall 30-day mortality was $3.4 \%$. The mortality rate for lobectomy/ bilobectomy, wedge resection, extended lobectomy/ bilobectomy and pneumonectomy was $2.6 \%, 4.2 \%, 4 \%$ and $8.5 \%$, respectively. ${ }^{20}$ In our study, in-hospital mortality was
2\% (post pneumonectomy: 5.5\%; post lobectomy: $1.5 \%$ and post sublobar resection: $0 \%$ )

Though VATS lobectomy has already established itself as the preferred surgical modality, we could perform it only in 9 patients $(5.7 \%)$. Two meta-analyses and two systematic reviews revealed that patients undergoing VATS lobectomy have reduced perioperative morbidity, mortality and less postoperative pain as short-term benefits compared to patients receiving open surgical resection while the long-term results (survival and recurrence rates) were not significantly different between the two surgical procedures. ${ }^{21-23}$ Whitson et al. with more than 6000 NSCLC patients undergoing either VATS or standard open lobectomy, showed not only that VATS lobectomy had fewer complications $138 \%$ vs. $44 \%$ ) and a shorter length of hospital stay (5 d vs. 7 d) compared to open surgical resection but rather that patients undergoing VATS at high volume hospitals had a shorter median length of hospital stay ( $4 \mathrm{~d}$ vs. 6 d) compared with patients receiving surgical resection at low volume hospitals. ${ }^{23}$ The main reason for less frequent VATS surgery in our series was learning curve for minimally invasive surgery and financial constraints from the patient's side (too expensive for the patient to afford for several staplers).

In our series, final pathological reports showed locally advanced stage (pIIIA-IIIB) in almost $50 \%$ of patients though the recommended neoadjuvant preoperative treatment was done only in $12 \%$ cases. The later could be the reason for poor overall 5 -year survival of $18 \%$. This again emphasizes the need of better preoperative diagnostic work up of mediastinal nodes, which was indeed suboptimal in our series.

Complete resection (RO) of non-small-cell lung cancer (NSCLC) is established as a necessary factor for potential long-term survival of patients undergoing surgery for NSCLC. ${ }^{24}$ Similarly N2 disease and the final stage of the disease are two other important factors influencing survival. The survival of cN2 patients with pathologically confirmed N2 (pN2) after surgery alone has been reported to be dismal $(10-15 \%$ of 5 -year overall survival). ${ }^{10}$ Our series also showed statistically significant survival favoring $\mathrm{pl}-\mathrm{Il}$ stages, RO resection and pNo-1 disease.

Therefore, we would like to conclude, based on the findings from surgical results of our study, that earlier stage (pl-II), RO resection and pathological pNo-1 has the best five year overall survival in Nepalese patients with NSCLC as well. 